\title{
Comparative study of preceding Campylobacter jejuni infection in Guillain-Barré syndrome in Japan and The Netherlands
}

\author{
M Koga, C W Ang, N Yuki, B C Jacobs, P Herbrink, F G A van der Meché, K Hirata, \\ $\mathrm{P} A$ van Doorn
}

Department of

Neurology, Dokkyo

University School of Medicine,

Kitakobayashi 880,

Mibu, Shimotsuga,

Tochigi 321-0293,

Japan

M Koga

N Yuki

K Hirata

Department of Neurology, Erasmus

University Medical

Centre Rotterdam,

Rotterdam, The

Netherlands

C W Ang

B C Jacobs

F G A van der Meché

P A van Doorn

Department of Immunology

C W Ang

B C Jacobs

Department of Immunology and Infectious Diseases, Diagnostic Centre SSDZ, Delft, The Netherlands

P Herbrink

Correspondence to: Dr N Yuki

yuki@dokkyomed.ac.jp

Received 25 September 2000 and in revised form

20 December 2000

Accepted 5 January 2001

\begin{abstract}
A comparative study was made in Japan and The Netherlands of the presence of preceding Campylobacter jejuni infections in Guillain-Barré syndrome (GBS). It was conducted in two laboratories using different serological criteria. The Japanese results showed no significant difference in the frequency of $\boldsymbol{C}$ jejuni infection between the Japanese $(17 / 88,19 \%)$ and Dutch $(21 / 132,16 \%)$ patients with GBS. The Dutch investigation showed a higher frequency in Dutch patients $(45 / 132 ; 34 \%)$ than in Japanese patients(20/88; 23\%), but the difference did not reach significance. Although the frequencies of preceding $C$ jejuni infection have been reported to be higher in Asian countries than in western countries, the findings of this collaborative study show that the incidence of antecedent $C$ jejuni infection in GBS in Japan is not higher than in The Netherlands and that serological assays vary considerably between laboratories.
\end{abstract}

(f Neurol Neurosurg Psychiatry 2001;70:693-695)

Keywords: Guillain-Barré syndrome; Campylobacter jejuni; enzyme linked immunosorbent assay

Guillain-Barré syndrome (GBS) is an immune mediated neuropathy, which occurs world wide, affecting patients of all ages and both sexes. Two thirds of patients with GBS have histories of antecedent infectious illness, and the gram negative bacterium Campylobacter jejuni has emerged as the most common antecedent infectious agent in GBS. ${ }^{1}$ Serological studies showed that the frequency of prior $C$ jejuni infection in GBS ranges from $17 \%$ to $66 \%$, the frequencies in northern China $(66 \%)^{2}$ and Japan $(45 \%)^{3}$ being higher than those in western countries such as the United States $(17 \%, 36 \%),{ }^{45}$ the United Kingdom $(26 \%),{ }^{6}$ The Netherlands (32\%), ${ }^{7}$ Germany $(39 \%),{ }^{8}$ and Australia (38\%). ${ }^{9}$ The reports suggest that in Asian countries GBS is more closely associated with $C$ jejuni than it is in western countries. These studies, however, used different assay systems, making it difficult to compare the incidence of preceding $C$ jejuni infection in GBS. A cooperative project by
Dokkyo University and Erasmus University was planned to compare the frequencies of $C$ jejuni associated GBS in Japan and The Netherlands. Serological examinations of the incidence of antecedent $C$ jejuni infection in the same serum samples from Japanese and Dutch patients with GBS were made in two laboratories, after which the frequency of $C$ jejuni infection and the correlation between the two different serological assays were analyzed.

\section{Patients and methods}

PATIENTS

Pretreatment serum samples were obtained from patients with GBS in Japan and The Netherlands, all of whom met the established criteria for GBS. ${ }^{10}$ The samples were stored at $-80^{\circ} \mathrm{C}$ until used. The Japanese GBS group consisted of 88 consecutive patients who had been referred to the Neuroimmunological Laboratory at Dokkyo University in 1998, from university hospitals and district general hospitals throughout Japan. None of them had been included in our previous study. ${ }^{11}$ The Dutch patients consisted of 132 of 147 patients with GBS who had participated in the Dutch GBS trial, $^{12} 15$ patients being excluded because no serum was available. These patients did not differ from the others in clinical manifestations and the course of the disease. Control serum samples were available from patients with other neurological diseases (27 Japanese and 42 Dutch) and from healthy volunteers (56 Japanese and 30 Dutch). There were no significant differences in the ages and sex of the patients with GBS, other neurological diseases, or of the healthy controls in Japan and The Netherlands.

\section{FEYUNI SEROLOGY}

Antecedent $C$ jejuni infection was serologically examined in the two laboratories by enzyme linked immunosorbent assays (ELISAs) as reported elsewhere. ${ }^{11}{ }^{13}$ These ELISAs are routinely used in each country for testing prior $C$ jejuni infecion in GBS. The antigen protein for ELISA used at Dokkyo University (Japanese ELISA) ${ }^{11}$ was prepared from a $C$ jejuni O:19 strain isolated from a Japanese patient with GBS and used at $100 \mathrm{ng}$ protein/well. Serum was considered positive when anti- $C$ jejuni $\operatorname{IgG}$ 

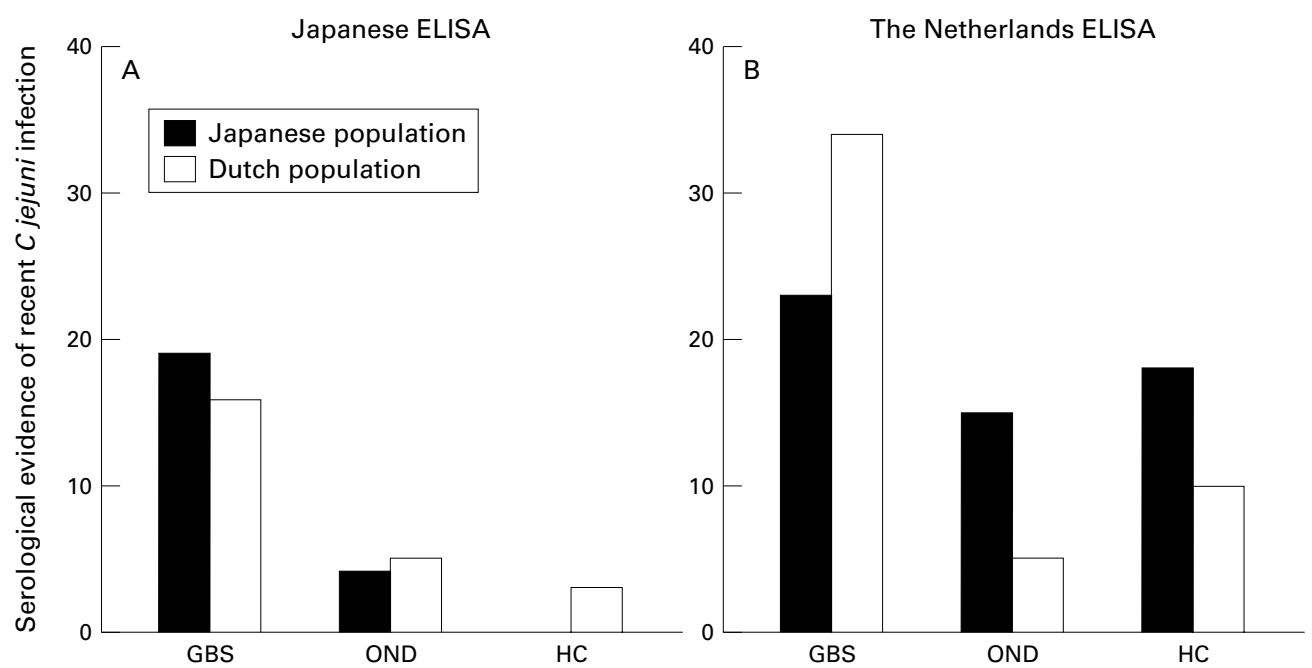

Figure 1 Frequency (\%) of serological evidence of recent $C$ jejuni infection in two laboratories: (A) fapan and (B) The Netherlands. Solid bar, Fapanese population; open bar, Dutch population; ELISA=enzyme linked immunosorbent assay; $G B S=$ Guillain-Barré syndrome; $O N D=$ other neurological diseases; $H C=$ healthy controls.

titres were 2000 or more in the Japanese ELISA. The cut off was defined based on the findings for 17 patients with GBS, from whom $C$ jejuni had been isolated, and findings for 46 healthy controls. This system has sensitivity of $82 \%$ and specificity of $88 \%$, and the presence of anti-C jejuni IgG antibody alone provided sufficient evidence of recent $C$ jejuni infection. ${ }^{11}$ Serum samples from the Japanese subjects were first used for the Japanese ELISA then immediately frozen at $-80^{\circ} \mathrm{C}$ and with ample dry ice air lifted to The Netherlands for testing by The Netherlands ELISA. ${ }^{13}$ Serum samples from Dutch subjects were treated similarly. In The Netherlands ELISA, the antigen protein was prepared from a $C$ jejuni Lau 48 strain isolated from a patient with enteritis and used at $300 \mathrm{ng}$ protein/well. Serological evidence of recent $C$ jejuni infection was defined as the presence of anti-C jejuni IgM, or IgA antibodies, or both. ${ }^{13}$ The Japanese and Dutch investigators were blinded to the diagnoses for each other's patients and serological results during the testing.

\section{STATISTICAL ANALYSIS}

The Spearman rank correlation test was used to test the association between the Japanese and Netherlands' ELISAs. The difference in the frequency of positive $C$ jejuni serology between these ELISAs was tested by the Mantel-Haenszel procedure. Other differences between groups were examined by the $\chi^{2}$ or Fisher's exact test where appropriate. Differences in medians were examined by the MannWhitney $U$ test. A difference was considered significant at $\mathrm{p}<0.05$. All statistical analyses were made with Statcel ${ }^{\circledR}$ software (OMS, Saitama, Japan).

\section{Results}

JAPANESE ELISA

Serological evidence of recent $C$ jejuni infection was found in $17(19 \%)$ of the 88 Japanese patients with GBS and $21(16 \%)$ of the 132 Dutch patients with GBS (fig 1). There was no significant difference between the Japanese and
Dutch patients with GBS, those with other neurological diseases, or healthy controls. In both the Japanese and Dutch populations, the frequency of positive $C$ jejuni serology was higher in the patients with GBS than in the patients with other neurological diseases or in the healthy controls, but this difference was significant only for Japanese patients with GBS and the healthy controls $(\mathrm{p}=0.0001)$.

\section{NETHERLANDS ELISA}

The incidence of positive $C$ jejuni serology in the Dutch patients with GBS (45/132; 34\%) was higher than in the Japanese patients with GBS (20/88; 23\%) ( $p=0.07)$ (fig 1). No significant differences were found between the Japanese $(15 \%)$ and Dutch $(5 \%)$ patients with other neurological diseases or between the Japanese (18\%) and Dutch (10\%) healthy controls. Dutch patients with GBS had positive $C$ jejuni serology significantly more often than the healthy controls $(p=0.009)$ and those who had other neurological diseases $(p=0.0002)$, whereas in the Japanese population, there was only a slight, non-significant difference in $C$ jejuni serology among the patients with GBS $(23 \%)$, those with other neurological diseases $(15 \%)$, and the healthy controls $(18 \%)$.

COMPARISON OF ELISA SYSTEMS

$C$ jejuni serology results showed good correlations in the Japanese $(\mathrm{p}<0.0001, r=0.77)$ and Dutch $(\mathrm{p}<0.0001, r=0.76)$ populations. Opposite results for both ELISAs were seen in 64 $(17 \%)$ of 375 serum samples included in this study (table 1). Most of these samples were positive in The Netherlands ELISA but

Table 1 Comparison of $C$ jejuni serology results from the two laboratories

\begin{tabular}{llr}
\hline & \multicolumn{2}{l}{ Fapanese ELISA } \\
\cline { 2 - 3 } & Positive & \multicolumn{1}{c}{ Negative } \\
\hline The Netherlands ELISA: & $31(8 \%)$ & $53(14 \%)$ \\
Positive & $11(3 \%)$ & $280(74 \%)$ \\
Negative & \\
\hline
\end{tabular}

ELISA=Enzyme linked immunosorbent assay. 
negative in the Japanese assay. The frequency of positive results was higher in The Netherlands $(22 \%)$ than in the Japanese ELISA $(11 \%)(\mathrm{p}=0.00003)$.

\section{Discussion}

Isolation of $C$ jejuni from the faeces of a patient is the most specific method for determining $C$ jejuni enteritis. Because GBS develops only 1-3 weeks after diarrhoea and the excretion period of the bacteria is limited, by this criterion a large proportion of patients with GBS related to $C$ jejuni would be negative. The incidence of antecedent gastrointestinal symptoms does not always reflect the frequency of preceding $C$ jejuni infection in GBS because asymptomatic $C$ jejuni infections are frequent and probably differ by country. ${ }^{14}$ By contrast, serodiagnostic methods are suitable for investigating preceding $C$ jejuni infection between countries.

We performed serological assays on the same serum samples in two widely separated laboratories. Both ELISA systems showed that the incidence of $C$ jejuni serology does not differ in Japanese and Dutch patients with GBS, but the true incidence of preceding $C$ jejuni infection in GBS is still not clear. Based on Japanese ELISA results, we reported elsewhere a $31 \%$ frequency of antecedent $C$ jejuni infection in Japanese patients with GBS, ${ }^{11}$ higher than that found in the present study. Every assay was standardised using well characterised serum with high anti-C jejuni antibody activity, and this discrepancy therefore may be due to the difference in patient populations surveyed in the previous and present studies. Because the population in the present study consisted of consecutive patients, we think that the Japanese patients in the present study are representative of the total group of Japanese patients with GBS.

Results of the assay systems routinely used in the two countries' laboratories were well correlated. The Netherlands ELISA, however, showed a higher frequency of positive results. Because all 11 Japanese patients with GBS from whom $C$ jejuni had been isolated were seropositive in The Netherlands ELISA (not included in this study), this discrepancy seems to reflect the different definition of positive serology, not the difference of the antigen preparation. In the Dutch patients with GBS, in particular, there was a great difference in seropositive frequency (34\% in The Netherlands' ELISA, $16 \%$ in the Japanese ELISA). This indicates that the incidence of preceding $C$ jejuni infection in GBS varies markedly with the assay's sensitivity and that it is difficult to compare the incidences of $C$ jejuni related GBS that were obtained by serological methods in previous studies. It is noteworthy that a high frequency of positive serology was found for Japanese patients with other neurological diseases $(15 \%)$ and the healthy controls (18\%) by The Netherlands ELISA, indicative that specificity of an assay used to determine $C$ jejuni serology may depend not only on the antigen preparation used but on the origin of the test population.

In conclusion, our comparative study showed that there is no significant difference in the incidence of preceding $C$ jejuni infection in patients with GBS in Japan and in The Netherlands. Differences between laboratories indicate that any worldwide comparative investigation of $C$ jejuni serology should be done in one laboratory and that the assay methods should be improved for yielding higher sensitivity and more specificity irrespective of the laboratory.

We thank Ms Y Tsuchiya and Mr M A de Klerk for their technical assistance. This research was supported in part by a grant from the Japanese Foundation for Neuroscience and Mental Health, grants in aid from the Ryoichi Naito Foundation for Medical Research, grants in aid for Scientific Research (10780482 and 10557063 to NY) from the Ministry of Education, Science, Culture, and Sports of Japan, a research grant for neuroimmunological diseases from the Ministry of Health and Welfare of Japan, and a grant from the Prinses Beatrix Fonds (No 95-0518). MK is a Research Fellow of the Japan Society for the Promotion of Science.

1 Jacobs BC, Rothbarth PH, van der Meché FGA, et al. The spectrum of antecedent infections in Guillain-Barré syndrome: a case-control study. Neurology 1998;51:1110-5.

2 Ho TW, Mishu B, Li CY, et al. Guillain-Barré syndrome in northern China: relationship to Campylobacter jejuni infection and anti-glycolipid antibodies Brain 1995;118: $597-605$

3 Hao Q, Saida T, Kuroki S, et al. Antibodies to gangliosides and galactocerebroside in patients with Guillain-Barré syndrome with preceding Campylobacter jejuni and other identified infections. F Neuroimmunol 1998;81:116-26.

4 Gruenewald R, Ropper AH, Lior $\mathrm{H}$, et al. Serologic evidence of Campylobacter jejuni/coli enteritis in patients with Guillain-Barré syndrome. Arch Neurol 1991;48:10802.

5 Mishu B, Ilyas AA, Koski CL, et al. Serologic evidence of previous Campylobacter jejuni infection in patients with the Guillain-Barré syndrome. Ann Intern Med 1993;118: 947-53.

6 Rees JH, Soudain SE, Gregson NA, et al. Campylobacter jejuni infection and Guillain-Barré syndrome. N Engl f Med 1995;333:1374-9.

7 Jacobs BC, van Doorn PA, Schmitz PIM, et al. Campylobacter jejuni infections and anti-GM1 antibodies in

8 Enders U, Karch H, Toyka KV, et al. The spectrum of immune responses to Campylobacter jejuni and glycoconugates in Guillain-Barré syndrome and in other neuroimmunological disorders. Ann Neurol 1993;34:136-44.

9 Kaldor J, Speed BR. Guillain-Barré syndrome and Campylobacter jejuni: a serological study. BMf 1984;288:186770 .

10 Asbury AK, Cornblath DR. Assessment of current diagnostic criteria for Guillain-Barré syndrome. Ann Neurol 1990; 27(suppl):S21-4.

11 Koga M, Yuki N, Takahashi $\mathrm{M}$, et al. Close association of IgA anti-ganglioside antibodies with antecedent Campylosyndromes. F Neuroimmunol 1998;81:138-43.

12 van der Meché FGA, Schmitz PIM, the Dutch GuillainBarré study group. A randomized trial comparing intravenous immune globulin and plasma exchange in GuillainBarré syndrome. N Engl f Med 1992;326:1123-9.

13 Herbrink P, van den Munckhof HA, Bumkens M, et al. Human serum antibody response in Campylobacter jejuni enteritis as measured by enzyme-linked immunosorbent assay. Eur F Clin Microbiol Infect Dis 1988;7:388-93.

14 Blaser MJ, Reller LB. Campylobacter enteritis. $N$ Engl $f$ Med 1981;305:1444-52. 